\title{
Admission oxygen saturation and all-cause in-hospital mortality in acute myocardial infarction patients: data from the MIMIC-III database
}

\author{
Yue Yu ${ }^{1 \#}$, Jun Wang ${ }^{1 \#}$, Qing Wang ${ }^{2 \#}$, Junnan Wang ${ }^{1,3 \#}$, Jie Min ${ }^{4}$, Suyu Wang ${ }^{1}$, Pei Wang ${ }^{1}$, Renhong Huang ${ }^{5}$, \\ Jian Xiao ${ }^{1}$, Yufeng Zhang ${ }^{1}$, Zhinong Wang ${ }^{1}$ \\ ${ }^{1}$ Department of Cardiothoracic Surgery, Changzheng Hospital, Naval Medical University, Shanghai, China; ${ }^{2}$ Department of Thoracic Surgery, Renji \\ Hospital, Shanghai Jiaotong University School of Medicine, Shanghai, China; ${ }^{3}$ Medical Research Center of War Injuries and Trauma, Changzheng \\ Hospital, Naval Medical University, Shanghai, China; ${ }^{4}$ Bethune International Peace Hospital, Shijiazhuang, China; ${ }^{5}$ Department of General Surgery, \\ Changzheng Hospital, Naval Medical University, Shanghai, China \\ Contributions: (I) Conception and design: Y Zhang, Z Wang, Y Yu; (II) Administrative support: Y Zhang, Z Wang, J Xiao; (III) Provision of study \\ materials or patients: None; (IV) Collection and assembly of data: Y Yu, J Wang, Q Wang, J Min, J Wang; (V) Data analysis and interpretation: Y Yu, \\ S Wang, P Wang, R Huang; (VI) Manuscript writing: All authors; (VII) Final approval of manuscript: All authors. \\ \#These authors contributed equally to this work. \\ Correspondence to: Yufeng Zhang, MD, PhD; Zhinong Wang, MD, PhD. Department of Cardiothoracic Surgery, Changzheng Hospital, Naval \\ Medical University, No. 415 Fengyang Road, Huangpu District, Shanghai, China. Email: zyflwj@smmu.edu.cn; wangzn007@smmu.edu.cn.
}

Background: Acute myocardial infarction (AMI) is mainly caused by a mismatch of blood oxygen supply and demand in the myocardium. However, several studies have suggested that excessively high or low arterial oxygen tension could have deleterious effects on the prognosis of AMI patients. Therefore, the relationship between blood oxygenation and clinical outcomes among AMI patients is unclear, and could be nonlinear. In the critical care setting, blood oxygen level is commonly measured continuously using pulse oximetryderived oxygen saturation $\left(\mathrm{SpO}_{2}\right)$. The present study aimed to determine the association between admission $\mathrm{SpO}_{2}$ levels and all-cause in-hospital mortality, and to elucidate the optimal $\mathrm{SpO}_{2}$ range with real-world data. Methods: Patients diagnosed with AMI on admission in the Medical Information Mart for Intensive Care III (MIMIC-III) database were included. A generalized additive model (GAM) with loess smoothing functions was used to determine and visualize the nonlinear relationship between admission $\mathrm{SpO}_{2}$ levels within the first 24 hours after ICU admission and mortality. Moreover, the Cox regression model was constructed to confirm the association between $\mathrm{SpO}_{2}$ and mortality.

Results: We included 1,846 patients who fulfilled our inclusion criteria, among whom 587 (31.80\%) died during hospitalization. The GAM showed that the relationship between admission $\mathrm{SpO}_{2}$ levels and all-cause in-hospital mortality among AMI patients was nonlinear, as a U-shaped curve was observed. In addition, the lowest mortality was observed for an $\mathrm{SpO}_{2}$ range of 94-96\%. Adjusted multivariable Cox regression analysis confirmed that the admission $\mathrm{SpO}_{2}$ level of 94-96\% was independently associated with decreased mortality compared to $\mathrm{SpO}_{2}$ levels <94\% [hazard ratio (HR) 1.352; 95\% confidence interval (CI): 1.048-1.715; $\mathrm{P}=0.028]$ and $>96 \%$ (HR 1.315; 95\% CI: 1.018-1.658; $\mathrm{P}=0.030$ ).

Conclusions: The relationship between admission $\mathrm{SpO}_{2}$ levels and all-cause in-hospital mortality followed a U-shaped curve among patients with AMI. The optimal oxygen saturation range was identified as an $\mathrm{SpO}_{2}$ range of $94-96 \%$, which was independently associated with increased survival in a large and heterogeneous cohort of AMI patients.

Keywords: Acute myocardial infarction (AMI); blood oxygen saturation; hospital mortality; hyperoxemia; oxygen therapy 
Submitted Mar 17, 2020. Accepted for publication Sep 14, 2020.

doi: $10.21037 /$ atm-20-2614

View this article at: http://dx.doi.org/10.21037/atm-20-2614

\section{Introduction}

Acute myocardial infarction (AMI) is a leading cause of mortality worldwide, which accounts for nearly 1.8 million deaths annually, and $20 \%$ of all deaths in Europe $(1,2)$. AMI mainly results from a mismatch of blood oxygen supply and demand in the myocardium that leads to ischemia and eventual cellular death (3). Therefore, supplemental oxygen to increase oxygen delivery to the ischemic myocardium has been routinely used in the treatment of AMI patients for over 100 years (4). However, excessively high oxygen tension might cause coronary vasoconstriction and increase the production of reactive oxygen species (ROS), potentially contributing to reperfusion injury $(5,6)$. Hence, blood oxygenation and mortality among AMI patients could be nonlinear and have a U-shaped relation. However, few empirical studies directly support this theory. Considering the high incidence and poor prognosis of AMI, it is necessary to determine the relationship between blood oxygenation and mortality, and explore its impact on survival, which could help more precisely predict the prognosis of AMI patients, and improve the implementation of appropriate oxygen therapy.

In critically ill patients with cardiorespiratory compromise, the blood oxygen level is commonly measured continuously using pulse oximetry-derived oxygen saturation $\left(\mathrm{SpO}_{2}\right)$, which could provide an early warning of hypoxemia $(7,8)$. Interestingly, a study showed that among AMI patients with normal peripheral oxygen saturations, lownormal oxygen saturation $\left(90 \% \leq \mathrm{SpO}_{2} \leq 94 \%\right)$ was identified as an independent marker of poor prognosis compared to high-normal oxygen saturation $\left(95 \% \leq \mathrm{SpO}_{2} \leq 100 \%\right)(9)$. However, the authors of that study classified $\mathrm{SpO}_{2}$ in arbitrarily defined categories rather than as a continuous variable, and did not explore the nonlinear relationship between $\mathrm{SpO}_{2}$ and clinical outcomes. Moreover, there are few guideline recommendations on the optimal oxygenation target specifically for AMI patients, and the available evidence to support such recommendations is limited $(1,10)$.

In this study, we aimed to determine the nonlinear relationship between admission $\mathrm{SpO}_{2}$ levels and all-cause inhospital mortality among patients with AMI, and to derive an optimal range of oxygen saturation for clinical practice and future research.

We present the following article in accordance with the Strengthening the Reporting of Observational Studies in Epidemiology (STROBE) reporting checklist (available at http://dx.doi.org/10.21037/atm-20-2614).

\section{Methods}

\section{Data source \& ethical statement}

All the relevant data were collected from the Medical Information Mart for Intensive Care-III (MIMIC-III) database. MIMIC-III is a freely accessible critical care database covering more than 50,000 hospital admissions comprised of 38,645 adults as well as 7,875 neonates admitted to surgical, trauma surgery, coronary, and cardiac surgery recovery intensive care units (ICUs) of Beth Israel Deaconess Medical Center (BIDMC) in Boston from 2001 to $2012(11,12)$. The MIMIC-III database documents included charted events such as demographic data, vital signs, laboratory findings and blood gas analysis data, prognostic scoring systems, and survival data. International Classification of Diseases, Ninth Revision (ICD-9) codes were recorded by hospital staff on patient discharge. Physiologic readings from bedside monitors were validated and documented hourly by ICU nurses. We passed the "Protecting Human Research Participants" exam and obtained permission to access the dataset (authorization code: 33281932). The establishment of the MIMIC-III database was approved by the Institutional Review Boards (IRB) of the Massachusetts Institute of Technology (MIT, Cambridge, MA, USA) and BIDMC. Our study utilized the anonymous data available from this database; hence, the requirement for informed consent was waived. In summary, the study complied with the ethical standards laid down in the 1964 Declaration of Helsinki and its later amendments.

\section{Population selection}

We included all ICU patients (aged $>18$ years) diagnosed with AMI using International Classification of Diseases (ICD)-9 diagnosis codes between 410.00 and 410.92 in the MIMIC-III database. Eligible patients had to have 
typical symptoms suggestive of MI (defined as chest pain or dyspnea) for $<6$ hours and either ischemic changes on electrocardiography or elevated cardiac troponin on admission (above the locally defined decision limit for MI) based on the third universal definition of AMI (13). Patients were excluded meeting the following criteria: (I) who had multiple admissions other than the first admission; (II) who had a secondary diagnosis of cancer, anemia, fluid and electrolyte disorder, or peripheral vascular disease (PVD) on admission; (III) who were admitted to the ICU during pregnancy, childbirth, or puerperium; (IV) who were at risk of oxygen-induced hypercapnia [chronic obstructive pulmonary disease (COPD), asthma, or pneumonia] on admission; (V) who stayed in the ICU less than 24 hours; (VI) who had incomplete or unobtainable documented $\mathrm{SpO}_{2}$ or other important medical data records.

\section{Data extraction and data processing}

The data were extracted from the database using structure query language (SQL) with PostgreSQL (version 9.4.6, www.postgresql.org). The code that supports the MIMICIII documentation and website is publicly available, and contributions from the community of users are encouraged (https://github.com/MIT-LCP/mimic-website). The variables in this study included demographics, admission type, vital signs, comorbidities, laboratory parameters, scoring systems, and clinical outcomes. Demographic information included age, gender, ethnicity, insurance status, marital status, and body mass index (BMI). BMI was calculated as weight $(\mathrm{kg})$ divided by height ${ }^{2}\left(\mathrm{~m}^{2}\right)$, using the height and weight reported at the time of admission. Vital signs included body temperature $(\mathrm{T})$, systolic blood pressure (SBP), diastolic blood pressure (DBP), mean blood pressure (MBP), heart rate (HR), respiratory rate (RR), and $\mathrm{SpO}_{2}$. Vital signs were measured multiple times within the first 24 hours after ICU admission, and the average values were used in our analysis as a measure of the central tendency of patients' conditions. Comorbidities included congestive heart failure (CHF), hypertension, cardiac arrhythmia, cardiogenic shock, cardiac arrest, pulmonary circulation disorder, diabetes mellitus (DM), renal failure, liver disease, coagulopathy, stroke, obesity, and weight loss. Moreover, the Elixhauser comorbidity index (ECI) was calculated to comprehensively evaluate the impact of comorbidities (14). Laboratory parameters included white blood cell (WBC) count, hematocrit (HCT), hemoglobin (Hb), blood platelet (PLT) count, glucose, cardiac troponin t, blood urea nitrogen (BUN), creatinine, and $\mathrm{PH}$. If patients received a laboratory test more than one time during their hospitalization, only the initial test results were included. Four scoring systems [the Glasgow Coma Scale (GCS), the Sequential Organ Failure Assessment (SOFA), the Systemic Inflammatory Response Syndrome (SIRS), and the Simplified Acute Physiology Score II (SAPS II)] were calculated within an hourly sliding 24-hour window, and the maximum was selected using the SQL code. For treatment information, oxygen therapy could refer to any oxygen supplementation methods such as face mask, non-invasive ventilation, or mechanical ventilation.

As extensive missing data might lead to bias, variables with over $30 \%$ missing values were not included in the subsequent analyses. Correspondingly, multivariate imputation (MI) was used for variables with less than $30 \%$ missing values $(15,16)$.

The endpoint of our study was all-cause in-hospital mortality, which was defined as survival status at hospital discharge. Patients with missing survival outcome data were excluded from the final cohort.

\section{Statistical analysis}

Baseline characteristics of enrolled participants were presented and compared between survivors and nonsurvivors by using either Student $t$-test, Kruskal Wallis rank test, Pearson's $\chi^{2}$ test, or Fisher's exact test as appropriate. Continuous variables were characterized as mean [standardized differences (SD) or median (interquartile range (IQR)], while categorical or ranked data were presented as count and proportion.

A generalized additive model (GAM) with loess smoothing functions was used to identify the nonlinear relationship between admission $\mathrm{SpO}_{2}$ readings and inhospital mortality. According to the results, an optimal oxygen saturation range was derived, and the study cohort was then divided into several subgroups with different $\mathrm{SpO}_{2}$ levels for subsequent analyses.

We also used Cox proportional hazards models to confirm the associations between $\mathrm{SpO}_{2}$ levels and mortality, with results expressed as hazard ratios (HRs) with $95 \%$ confidence intervals (CIs). A three-step Cox regression model was constructed based on different $\mathrm{SpO}_{2}$ groups. Model I included only the $\mathrm{SpO}_{2}$ data. In Model II, covariates were adjusted for age and gender. Model III further adjusted for covariates that were statistically significant $(\mathrm{P}<0.100)$ in the univariable Cox regression 
model. We tested for detrimental effects of collinearity on the model using variance inflation factors (VIFs) (17). The Kaplan-Meier (KM) method was used to plot unadjusted survival curves, and the log-rank test was used to compare differences between groups. A series of sensitivity analyses were performed to further validate the robustness of our findings.

A two-tailed $\mathrm{P}$ value of less than 0.050 was considered to be statistically significant. All statistical analyses were performed using SPSS software (version 22.0; IBM Corporation, St. Louis, Missouri, USA) and R software (version.3.6.1; The R Project for Statistical Computing, TX, USA; http://www.r-project.org).

\section{Results}

\section{Subject and variable characteristics}

After application of the inclusion and exclusion criteria, the final study cohort consisted of 1,846 AMI patients, of whom 587 (31.80\%) patients died during hospitalization. The detailed information on the enrollment and selection process was summarized in Table S1. In total, 42 variables were extracted from the MIMIC-III database, and 11 of them had missing values (Table S2).

The comparison of baseline characteristic between survivors and non-survivors was summarized in Table 1. Notably, patients of the non-survivor group were much older than those of the survivor group [75.00 (64.50-83.00) vs. 65.00 (56.00-77.00); $\mathrm{P}<0.001$ ], while more patients in the survivor group had higher BMI compared to those in the non-survivor group [27.67 (24.37-30.99) vs. 26.44 (23.05-30.15); $\mathrm{P}<0.001]$. As for the comorbidities, patients who died during hospitalization had higher incidence of CHF $(56.90 \%$ vs. $37.41 \% ; \mathrm{P}<0.001)$, cardiac arrhythmias (56.22\% vs. $45.04 \% ; \mathrm{P}<0.001)$, stroke $(13.63 \%$ vs. $5.48 \%$; $\mathrm{P}<0.001)$, cardiogenic shock $(32.54 \%$ vs. $7.15 \%)$, cardiac arrest (15.50\% vs. $3.73 \%)$, renal failure $(20.44 \%$ vs. $9.93 \%$; $\mathrm{P}<0.001)$, liver disease $(7.33 \%$ vs. $2.86 \% ; \mathrm{P}<0.001)$, and coagulopathy $(11.41 \%$ vs. $6.67 \% ; \mathrm{P}<0.001)$. With regard to the vital signs, HR [84.71 (73.81-95.79) vs. 80.63 (71.05-89.94); $\mathrm{P}<0.001]$ and $\mathrm{RR}$ [18.89 (16.83-21.70) vs. 17.96 (16.09-20.05); $\mathrm{P}<0.001]$ were significantly higher in the non-survivor group, while the SBP [108.67 (98.94$119.78)$ vs. 112.30 (103.45-121.78); $\mathrm{P}<0.001]$, DBP [56.79 (50.14-63.94) vs. 60.40 (54.68-67.65); $\mathrm{P}<0.001], \mathrm{MBP}$ [74.09 (68.00-81.36) vs. 77.52 (71.88-83.88); $\mathrm{P}<0.001]$, PLT [231.00 (179.00-288.50) vs. 238.00 (185.00-305.08);
$\mathrm{P}=0.049]$ as well as $\mathrm{PH}[7.35$ (7.30-7.39) vs. 7.37 (7.327.43); $\mathrm{P}=0.039$ ] were lower than those from the survivor group. No difference was observed in admission $\mathrm{SpO}_{2}$ level between the two cohorts [95.57 (94.30-96.63) vs. 95.82 (94.98-96.79); $\mathrm{P}=0.621]$.

\section{Relationship between oxygen saturation and all-cause in- hospital mortality}

The relationship between admission $\mathrm{SpO}_{2}$ level and allcause in-hospital mortality was nonlinear, and a U-shaped curve was observed, as shown in Figure 1. While low $\mathrm{SpO}_{2}$ correlated more strongly with mortality, high $\mathrm{SpO}_{2}$ was also associated with increased mortality. Informed by the flattest part of the U-shape in Figure 1, we chose an $\mathrm{SpO}_{2}$ range of $94-96 \%$ as the optimal oxygen saturation range, and then divided the study cohort into three groups with different $\mathrm{SpO}_{2}$ levels: Group $1\left(94 \% \leq \mathrm{SpO}_{2} \leq 96 \%\right)$, Group $2\left(\mathrm{SpO}_{2}\right.$ $<94 \%)$, and Group $3\left(96 \%<\mathrm{SpO}_{2} \leq 100 \%\right)$.

\section{Survival analysis}

The unadjusted survival curve for patients with different $\mathrm{SpO}_{2}$ groups is shown in a Kaplan-Meier plot in Figure 2 (log-rank test: $\mathrm{P}<0.001)$. We used Cox regression models to determine the association between the different $\mathrm{SpO}_{2}$ groups and hospital mortality among patients with AMI. Group $1\left(94 \% \leq \mathrm{SpO}_{2} \leq 96 \%\right)$ was always considered as the reference group. Model I showed that Group 1 (94\% $\leq$ $\left.\mathrm{SpO}_{2} \leq 96 \%\right)$ was associated with decreased risk of allcause mortality compared to Group 2 (HR $=1.783 ; 95 \%$ CI: $1.433-2.217 ; \mathrm{P}<0.001)$ and Group 3 (HR $=1.495$; 95\% CI: 1.245-1.796; $\mathrm{P}<0.001$ ) (Table 2). After adjustment for age and gender, Model II showed similar results (Group 2: $\mathrm{HR}=1.859 ; 95 \%$ CI: 1.494-2.313; $\mathrm{P}<0.001 ;$ Group 3: $\mathrm{HR}=1.485 ; 95 \%$ CI: 1.237-1.784; $\mathrm{P}<0.001$ ) (Table 2). The univariable Cox regression analysis suggested that age, gender, marital status, admission type, DBP, MBP, RR, T, cardiogenic shock, cardiac arrest, renal failure, coagulopathy, weight loss, SOFA, SAPS II, percutaneous coronary intervention (PCI), ICU length of stay (LOS), and $\mathrm{SpO}_{2}$ level were potential prognostic factors for mortality in Table S3 (all $\mathrm{P}<0.100)$, which were then entered into the multivariable Cox regression model (Model III). Additionally, VIFs did not show any possibility of collinearity between $\mathrm{SpO}_{2}$ and the other variables in Model III (maximum VIF of 4.2, which is below the threshold of concern, VIF <5). Model III demonstrated that age 
Table 1 Baseline characteristics between survivors and non-survivors

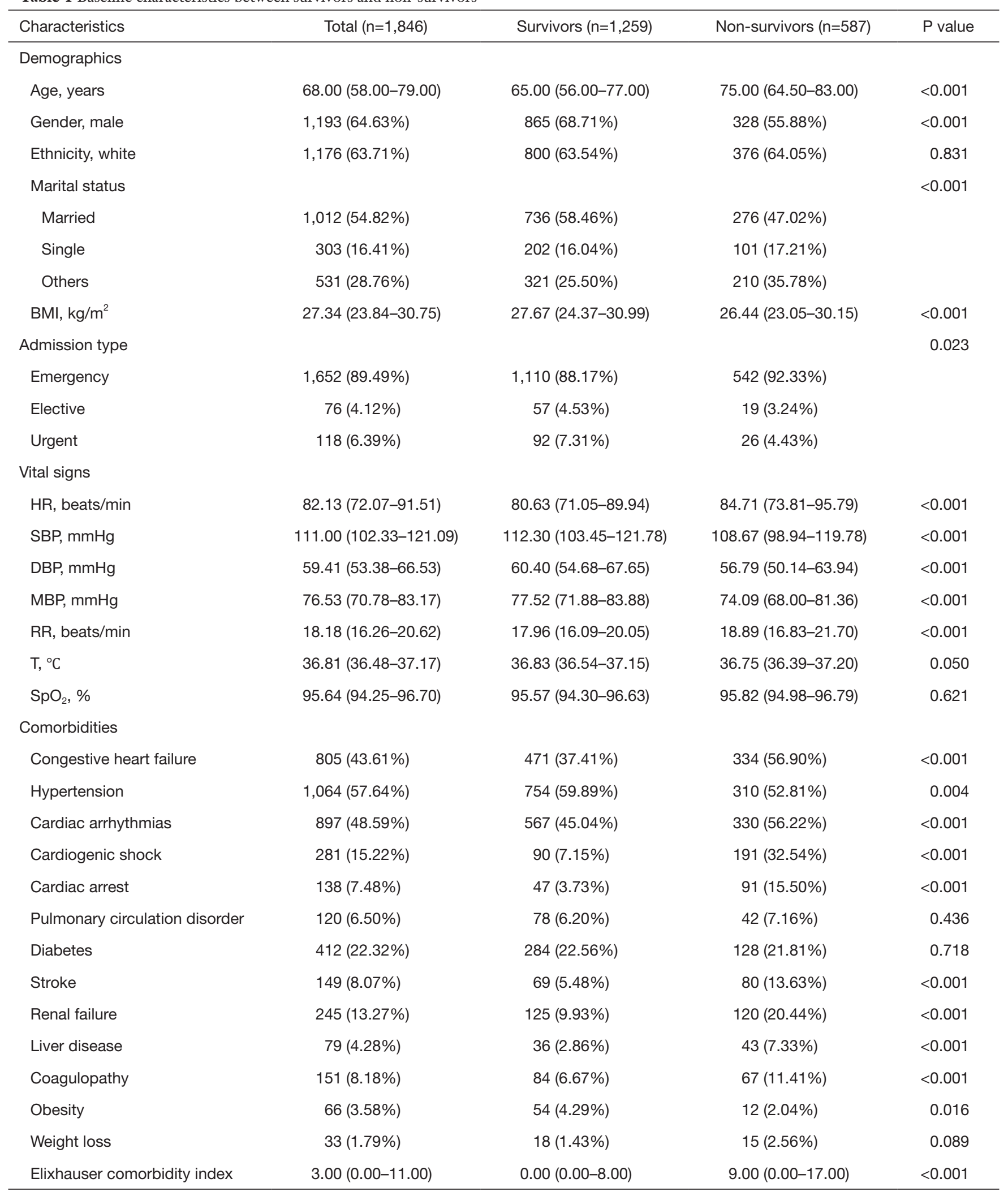

Table 1 (continued) 
Table 1 (continued)

\begin{tabular}{|c|c|c|c|c|}
\hline Characteristics & Total $(n=1,846)$ & Survivors $(n=1,259)$ & Non-survivors $(\mathrm{n}=587$ ) & $P$ value \\
\hline WBC, $10^{9} / \mathrm{L}$ & 11.60 (8.70-15.02) & $11.77(8.70-15.10)$ & $11.20(8.80-14.98)$ & 0.219 \\
\hline $\mathrm{Hb}, \mathrm{g} / \mathrm{dL}$ & $12.50(11.00-14.10)$ & $12.43(10.93-14.10)$ & $12.60(11.00-14.10)$ & 0.778 \\
\hline HCT, \% & $36.01(6.28)$ & $36.00(6.31)$ & $36.05(6.23)$ & 0.857 \\
\hline Troponin $\mathrm{t}, \mathrm{ng} / \mathrm{mL}$ & $1.26(0.06-4.73)$ & $1.31(0.07-4.66)$ & $1.10(0.03-4.84)$ & 0.420 \\
\hline BUN, mg/dL & $22.00(15.00-34.21)$ & $22.00(15.00-34.65)$ & $22.00(15.00-34.00)$ & 0.376 \\
\hline Glucose, mg/dL & $141.00(110.00-198.32)$ & $142.00(109.56-199.50)$ & $137.23(111.00-197.50)$ & 0.631 \\
\hline Creatinine, $\mathrm{mEq} / \mathrm{L}$ & $1.00(0.80-1.50)$ & $1.10(0.80-1.50)$ & $1.00(0.80-1.50)$ & 0.365 \\
\hline SAPS II & $33.00(25.00-44.00)$ & $30.00(23.00-39.00)$ & $42.00(32.50-53.00)$ & $<0.001$ \\
\hline SIRS & $3.00(2.00-4.00)$ & $3.00(2.00-3.00)$ & $3.00(2.00-4.00)$ & $<0.001$ \\
\hline GCS & $15.00(15.00-15.00)$ & $15.00(15.00-15.00)$ & $15.00(14.00-15.00)$ & $<0.001$ \\
\hline \multicolumn{5}{|l|}{ Treatment information } \\
\hline $\mathrm{PCl}$ & $1,199(64.95 \%)$ & 917 (72.84\%) & $282(48.04 \%)$ & $<0.001$ \\
\hline CABG & 59 (3.20\%) & $40(3.18 \%)$ & 19 (3.24\%) & 0.076 \\
\hline Oxygen therapy & $949(51.41 \%)$ & $663(52.66 \%)$ & $286(48.72 \%)$ & 0.115 \\
\hline
\end{tabular}

Values are $\mathrm{n}(\%)$, mean $\pm \mathrm{SD}$, or median (interquartile range). BMI, body mass index; HR, heart rate; SBP, systolic blood pressure; MBP, mean blood pressure; DBP, diastolic blood pressure; RR, respiratory rate; T, temperature; WBC, white blood cell; Hb, hemoglobin; HCT, hematocrit; PLT, platelet; BUN, blood urea nitrogen; SOFA, Sequential Organ Failure Assessment; SAPS, Systemic Inflammatory Response Syndrome; SIRS, Systemic Inflammatory Response Syndrome; GCS, Glasgow Coma Scale; PCl, percutaneous coronary intervention; CABG, coronary artery bypass grafting; LOS, length of stay.

$(\mathrm{HR}=1.012 ; 95 \%$ CI: $1.002-1.018 ; \mathrm{P}=0.001), \mathrm{DBP}(\mathrm{HR}$ $=0.954 ; 95 \%$ CI: $0.933-0.981 ; \mathrm{P}=0.003)$, admission type $(\mathrm{HR}=0.511 ; 95 \%$ CI: $0.288-0.889 ; \mathrm{P}=0.041 ; \mathrm{HR}=0.581$; 95\% CI: 0.372-0.902; $\mathrm{P}=0.017)$, RR (HR $=1.052 ; 95 \%$ CI: $1.030-1.082 ; \mathrm{P}<0.001)$, cardiogenic shock $(\mathrm{HR}=1.711$; 95\% CI: $1.425-2.117 ; \mathrm{P}<0.001)$, cardiac arrest $(\mathrm{HR}=2.048$; 95\% CI: $1.674-2.432 ; \mathrm{P}<0.001)$, SOFA (HR $=1.044 ; 95 \%$ CI: $1.001-1.087 ; \mathrm{P}=0.040)$, SAPS II (HR $=1.011 ; 95 \% \mathrm{CI}$ : $1.000-1.021$; $\mathrm{P}=0.018)$, PCI (HR $=0.722 ; 95 \%$ CI: $0.502-$ 0.789; $\mathrm{P}<0.001$ ), and $\mathrm{SpO}_{2}$ level (Group 2: $\mathrm{HR}=1.352 ; 95 \%$ CI: $1.048-1.715$; $\mathrm{P}=0.028$; Group 3: HR $=1.315 ; 95 \%$ CI: $1.018-1.658 ; \mathrm{P}=0.030)$ were all independent prognostic factors for predicting hospital mortality in patients with
AMI (Tables 2, Table S3).

\section{Sensitivity analyses}

A series of sensitivity analyses were performed to validate the robustness of our findings. First, we excluded 11 patients with hypoxemia $\left(\mathrm{SpO}_{2}<90 \%\right)$ and found Group $1(94 \% \leq$ $\mathrm{SpO}_{2} \leq 96 \%$ ) was an independent prognostic predictor even among patients without hypoxemia (Table S4). Second, we used the original data for analysis without using the MI method, and 1,049 patients remained in the final cohort. After adjustment for covariates in Model III, Group 1 (94\% $\leq$ $\mathrm{SpO}_{2} \leq 96 \%$ ) was not independently associated with hospital 

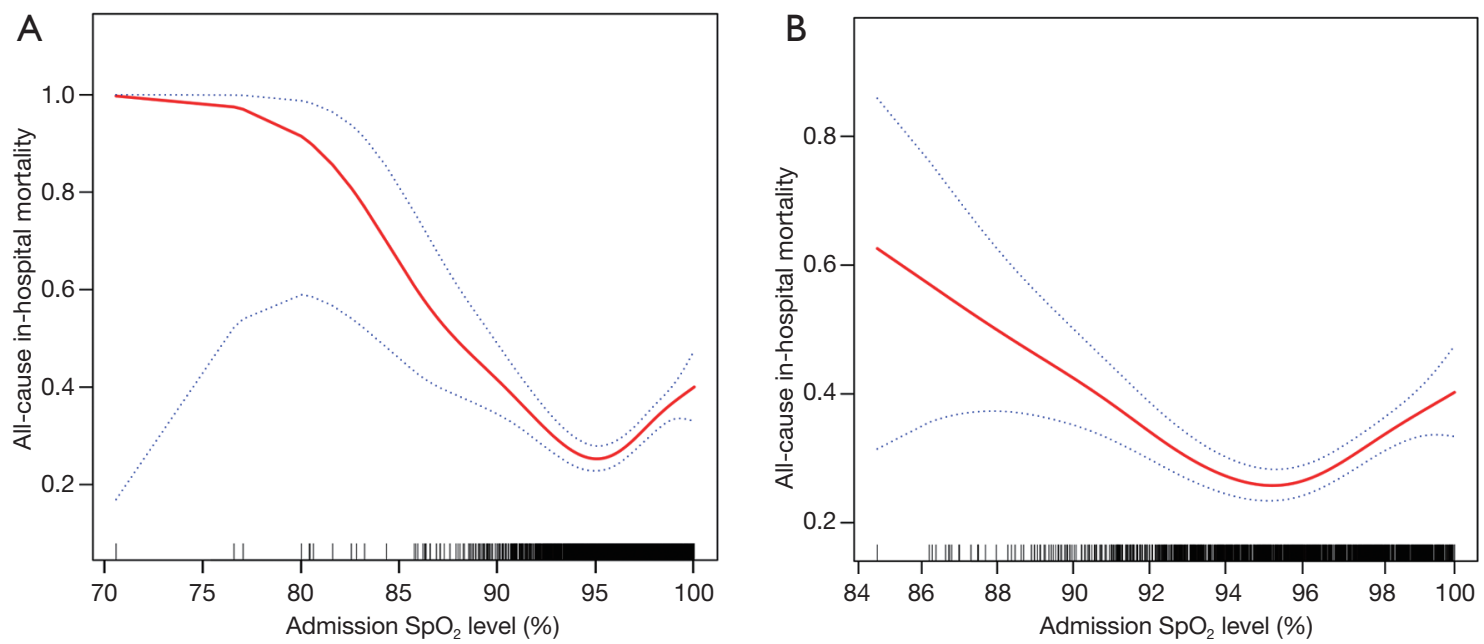

Figure 1 Relationship between admission $\mathrm{SpO}_{2}$ levels and mortality. (A) Showed the relationship between admission $\mathrm{SpO}_{2}$ levels and allcause in-hospital mortality in AMI patients by using a generalized additive model and (B) showed the U-shaped part of Figure 1A. Solid red line represents the smooth curve fit between variables. Dotted blue lines represent the $95 \%$ of confidence interval from the fit. AMI, acute myocardial infarction; $\mathrm{SpO}_{2}$, pulse oximetry-derived oxygen saturation.

mortality when compared to Group $3\left(96 \%<\mathrm{SpO}_{2} \leq 100 \%\right)$, which might have resulted from the reduction in the number of participants (Table S5). In addition, as shown in Table 3, the association between $\mathrm{SpO}_{2}$ and hospital mortality was similar for most strata except for some subgroups with small sample sizes. Among these strata, we observed that the $\mathrm{SpO}_{2}$ range of $94-96 \%$ had significantly lower mortality in patients with age $<65$ years, age $\geq 65$ years, male, female, married status, $\mathrm{BMI}<27 \mathrm{~kg} / \mathrm{m}^{2}, \mathrm{BMI} \geq 27 \mathrm{~kg} / \mathrm{m}^{2}$, admission type (Emergency), $\mathrm{HR}<82$ beats/min, $\mathrm{HR} \geq 82$ beats/min, SBP $<110 \mathrm{mmHg}, \mathrm{SBP} \geq 110 \mathrm{mmHg}, \mathrm{DBP}<60 \mathrm{mmHg}$, MBP $<75 \mathrm{mmHg}, \mathrm{MBP} \geq 75 \mathrm{mmHg}, \mathrm{RR} \geq 18$ beats/min, CHF, hypertension, cardiac arrhythmias, diabetes, SOFA $\geq 3$, SAPS II $\geq 33$, and oxygen therapy.

\section{Discussion}

In the current study, our analyses demonstrated a U-shaped relationship between early admission $\mathrm{SpO}_{2}$ readings and allcause in-hospital mortality among patients with AMI. In addition, the multivariable Cox regression analysis identified $\mathrm{SpO}_{2}$ as an independent prognostic predictor of clinical outcomes during hospitalization. Moreover, our study also showed the lowest mortality for an $\mathrm{SpO}_{2}$ range of 94-96\%, which could become the optimal oxygen saturation targets and benefit oxygen therapy among AMI patients. To our knowledge, this study was the first to explore the nonlinear relationship of admission $\mathrm{SpO}_{2}$ level and all-cause inhospital mortality among AMI patients.

Pulse oximetry is a ubiquitously used monitoring technique for patients in ICUs (7). Using a spectrophotometric methodology, pulse oximetry measures oxygen saturation by illuminating the skin and measuring changes in light absorption of oxygenated and deoxygenated blood using two light wavelengths: 660 and $940 \mathrm{~nm}(7,18,19)$. The ratio of absorbance at these two wavelengths is calibrated against direct measurements of arterial oxygen saturation $\left(\mathrm{SaO}_{2}\right)$ to calculate the pulse oximeter's measure of arterial saturation. $\mathrm{SpO}_{2}$ provides pragmatic advantages over the arterial partial pressure of oxygen $\left(\mathrm{PaO}_{2}\right)$ and $\mathrm{SaO}_{2}$, including the ability to inexpensively, noninvasively and repeatedly measure blood oxygenation (20). Additionally, $\mathrm{SpO}_{2}$ is also clinically more relevant as adjustments of inspired oxygen, and ventilator settings are based on $\mathrm{SpO}_{2}$ changes rather than on intermittent arterial blood gas assays. Therefore, it is common practice to use $\mathrm{SpO}_{2}$ as a surrogate for $\mathrm{SaO}_{2}$. The agreement between $\mathrm{SpO}_{2}$ and $\mathrm{SaO}_{2}$ is sufficient to use them interchangeably (mean difference 1 $\pm 2 \%$ ), and the specificity of the latest generation devices to detect hypoxemia is $>95 \%$ (21). Furthermore, using $\mathrm{SpO}_{2}$ to titrate supplemental oxygen is superior to fixed inspired oxygen fractions, which risk over-oxygenation in patients with narrow alveolar arterial oxygen gradients, and underoxygenation in those with broad gradients. However, due 
Group $+1 \div 2 \div 3$
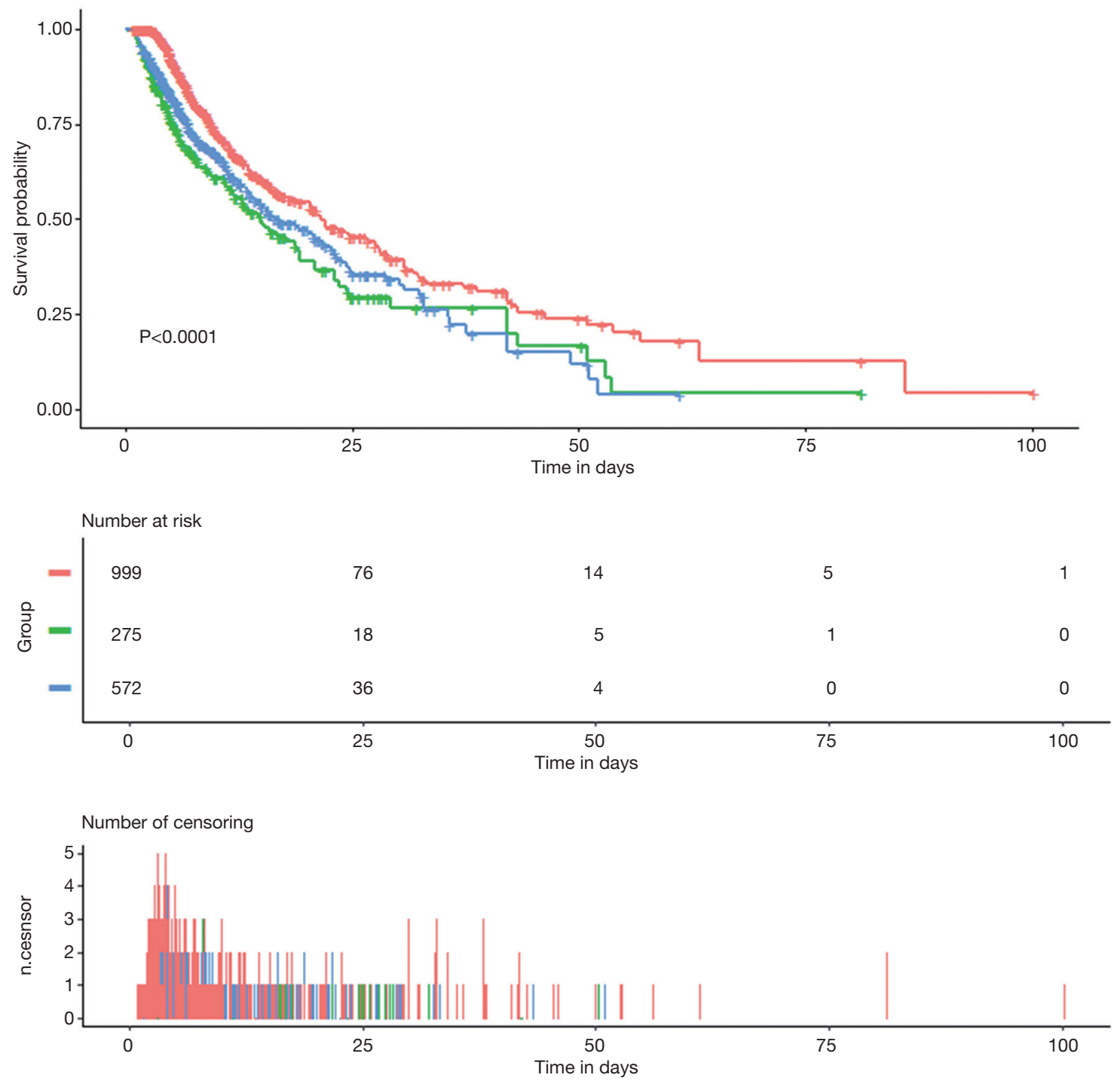

Figure 2 Kaplan-Meier plot for AMI patients with different $\mathrm{SpO}_{2}$ levels. Group 1 represents 94\% $\leq \mathrm{SpO}_{2} \leq 96 \%$. Group 2 represents $\mathrm{SpO}$, $<94 \%$. Group 3 represents $96 \%<\mathrm{SpO}_{2} \leq 100 \%$. AMI, acute myocardial infarction; $\mathrm{SpO}_{2}$, pulse oximetry-derived oxygen saturation.

Table 2 Relationship between $\mathrm{SpO}_{2}$ levels and all-cause in-hospital mortality in different Cox regression models

\begin{tabular}{|c|c|c|c|c|c|c|}
\hline Variables & \multicolumn{2}{|l|}{ Model I } & \multicolumn{2}{|l|}{ Model II } & \multicolumn{2}{|l|}{ Model III } \\
\hline \multicolumn{7}{|l|}{$\mathrm{SpO}_{2}$ groups } \\
\hline Group $1\left(94 \% \leq \mathrm{SpO}_{2} \leq 96 \%\right)$ & Ref & & Ref & & Ref & \\
\hline Group $2\left(\mathrm{SpO}_{2}<94 \%\right)$ & $1.783(1.433,2.217)$ & $<0.001$ & $1.859(1.494,2.313)$ & $<0.001$ & $1.352(1.048,1.715)$ & 0.028 \\
\hline
\end{tabular}

$\mathrm{HR}$, hazard ratio; $\mathrm{Cl}$, confidence interval; ref, reference. 
Table 3 Subgroup analysis of the relationship between $\mathrm{SpO}_{2}$ levels and all-cause in-hospital mortality

\begin{tabular}{|c|c|c|c|c|c|c|}
\hline Characteristics & $\mathrm{N}$ & $\begin{array}{c}\text { Group } 1 \\
\text { (Ref) }\end{array}$ & \multicolumn{2}{|l|}{ Group 2} & \multicolumn{2}{|l|}{ Group 3} \\
\hline \multicolumn{7}{|l|}{ Age, years } \\
\hline$<65$ & 745 & Ref & $1.972(1.265,3.075)$ & 0.003 & $2.020(1.395,2.926)$ & $<0.001$ \\
\hline$\geq 65$ & 1,101 & Ref & $1.779(1.383,2.287)$ & $<0.001$ & $1.358(1.099,1.678)$ & 0.005 \\
\hline Male & 1,193 & Ref & $1.434(1.065,1.931)$ & 0.018 & $1.358(1.065,1.733)$ & 0.014 \\
\hline Female & 653 & Ref & $2.445(1.764,3.387)$ & $<0.001$ & $1.696(1.280,2.246)$ & $<0.001$ \\
\hline \multicolumn{7}{|l|}{ Marital status } \\
\hline Married & 1,012 & Ref & $2.141(1.575,2.911)$ & $<0.001$ & $1.649(1.253,2.169)$ & $<0.001$ \\
\hline$<27$ & 948 & Ref & $1.808(1.352,2.418)$ & $<0.001$ & $1.457(1.144,1.855)$ & 0.002 \\
\hline$\geq 27$ & 898 & Ref & $1.699(1.220,2.365)$ & 0.002 & $1.508(1.136,2.001)$ & 0.004 \\
\hline \multicolumn{7}{|l|}{ Admission type } \\
\hline Emergency & 1,652 & Ref & $1.796(1.430,2.256)$ & $<0.001$ & $1.529(1.265,1.849)$ & $<0.001$ \\
\hline Elective & 76 & Ref & $1.022(0.312,3.349)$ & 0.971 & $0.758(0.232,2.476)$ & 0.647 \\
\hline Urgent & 118 & Ref & $3.064(1.107,8.479)$ & 0.031 & $1.282(0.505,3.255)$ & 0.602 \\
\hline \multicolumn{7}{|l|}{$\mathrm{HR}$, beats/min } \\
\hline \multicolumn{7}{|l|}{ DBP, $\mathrm{mmHg}$} \\
\hline$<60$ & 929 & Ref & $2.159(1.630,2.860)$ & $<0.001$ & $1.550(1.225,1.961)$ & $<0.001$ \\
\hline$\geq 60$ & 917 & Ref & $1.365(0.956,1.949)$ & 0.087 & $1.363(1.015,1.831)$ & 0.040 \\
\hline \multicolumn{7}{|l|}{ MBP, $\mathrm{mmHg}$} \\
\hline$<75$ & 755 & Ref & $1.851(1.378,2.488)$ & $<0.001$ & $1.512(1.167,1.957)$ & 0.002 \\
\hline$\geq 75$ & 1,091 & Ref & $1.601(1.149,2.230)$ & 0.005 & $1.436(1.106,1.864)$ & 0.007 \\
\hline \multicolumn{7}{|l|}{$\mathrm{RR}$, beats/min } \\
\hline$<18$ & 744 & Ref & $2.042(1.308,3.187)$ & 0.002 & $1.251(0.920,1.700)$ & 0.153 \\
\hline$\geq 18$ & 1,102 & Ref & $1.668(1.297,2.146)$ & $<0.001$ & $1.845(1.465,2.324)$ & $<0.001$ \\
\hline \multicolumn{7}{|c|}{ Congestive heart failure } \\
\hline No & 1,041 & Ref & $2.549(1.827,3.556)$ & $<0.001$ & $1.679(1.267,2.225)$ & $<0.001$ \\
\hline Yes & 805 & Ref & $1.392(1.040,1.863)$ & 0.026 & $1.388(1.088,1.771)$ & 0.008 \\
\hline
\end{tabular}

Table 3 (continued) 
Table 3 (continued)

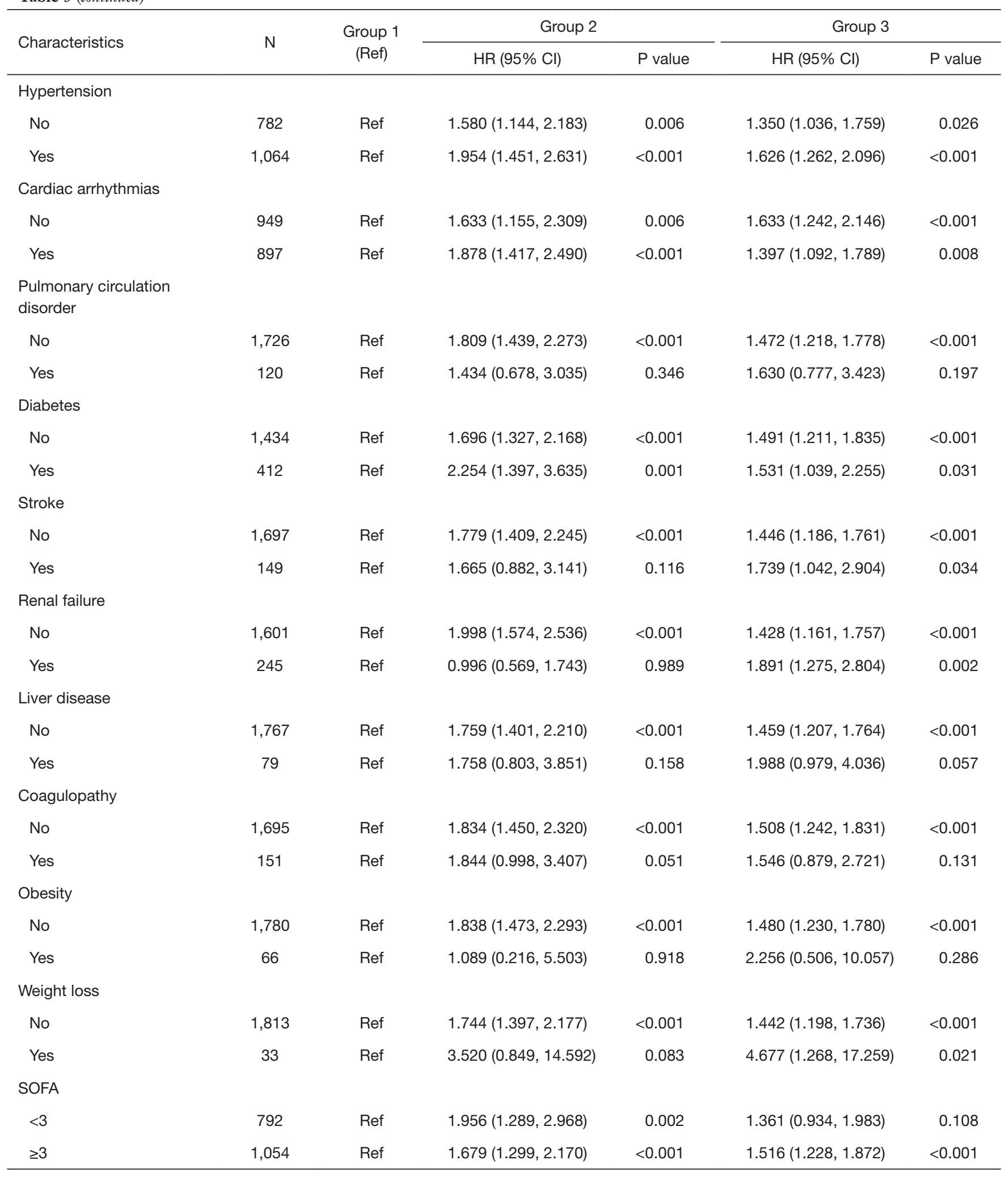

Table 3 (continued) 
Table 3 (continued)

\begin{tabular}{|c|c|c|c|c|c|c|}
\hline Characteristics & $\mathrm{N}$ & $\begin{array}{c}\text { Group } 1 \\
\text { (Ref) }\end{array}$ & \multicolumn{2}{|c|}{ Group 2} & \multicolumn{2}{|l|}{ Group 3} \\
\hline \multicolumn{7}{|l|}{ SAPS II } \\
\hline$<33$ & 885 & Ref & $1.401(0.922,2.128)$ & 0.114 & $1.213(0.827,1.781)$ & 0.323 \\
\hline$\geq 33$ & 961 & Ref & $1.992(1.541,2.575)$ & $<0.001$ & $1.555(1.259,1.922)$ & $<0.001$ \\
\hline No & 897 & Ref & $2.442(1.781,3.350)$ & $<0.001$ & $1.905(1.471,2.468)$ & $<0.001$ \\
\hline Yes & 949 & Ref & $1.343(0.104,1.823)$ & 0.048 & $1.194(0.108,1.570)$ & 0.050 \\
\hline \multicolumn{7}{|c|}{$\begin{array}{l}\text { Renal replacement } \\
\text { treatment }\end{array}$} \\
\hline
\end{tabular}

Covariates were adjusted as in Model I. N, number; BMI, body mass index; SBP, systolic blood pressure; MBP, mean blood pressure; DBP, diastolic blood pressure; RR, respiratory rate; SOFA, Sequential Organ Failure Assessment; SAPS, Systemic Inflammatory Response Syndrome; HR, hazard ratio; $\mathrm{Cl}$, confidence interval; ref, reference.

to the sigmoidal shape of the oxyhemoglobin dissociation curve, $\mathrm{SpO}_{2}$ may not detect hyperoxemia in patients with high $\mathrm{PaO}_{2}$ levels (7). However, for the $\mathrm{SpO}_{2}$ range of 94$96 \%$, the correlation between $\mathrm{SpO}_{2}$ and $\mathrm{PaO}_{2}$ would be fair, with little risk of underestimation of either hypoxemia or hyperoxemia $(22,23)$.

All patients with AMI should undergo an early assessment of short-term risk. Several risk scores such as the Global Registry of Acute Coronary Events (GRACE) risk score have been developed, based on readily identifiable parameters in the acute phase $(24,25)$. Blood oxygen saturation has been used as a useful prognostic predictor in many diseases (26-29). However, few studies have investigated the prognostic value of $\mathrm{SpO}_{2}$ levels among $\mathrm{AMI}$ patients. In the present study, the assessment of early $\mathrm{SpO}_{2}$ readings within the first 24 hours after patients' admission could serve as a preliminary prognostic marker for shortterm mortality even among normoxic patients, which could help distinguish low-risk and high-risk AMI cohort and tailor individualized treatment. Similar to our results, James et al. (9) found that the $\mathrm{SpO}_{2}$ range of 90-94\% was associated with poor clinical outcomes compared to the $\mathrm{SpO}_{2}$ range of $95-100 \%$ among patients with confirmed MI. However, considering the U-shaped relationship between $\mathrm{SpO}_{2}$ and mortality, perhaps three, rather than two, $\mathrm{SpO}_{2}$ groups are required to explore the effect of $\mathrm{SpO}_{2}$ on patients' prognoses. In addition, our study showed that two scoring systems (SOFA and SAPS II) provided potentially valuable prognostic information on clinical outcomes when applied to patients with AMI. Huang et al. found that the SOFA score and the GRACE score provided better discrimination for long-term mortality than did the thrombolysis in myocardial infarction (TIMI) score (30). Different from their results, we mainly focused on the prognostic value of the SOFA to predict short-term mortality. No previous study has reported the prognostic ability of the SAPS II score in AMI patients, and further investigation is required to confirm our findings.

Although oxygen therapy is a standard medical practice during AMI, there is no clear oxygen therapy guideline for AMI patients, which might be attributed to the lack of evidence on the optimal oxygenation target. Our study showed the lowest mortality at a $\mathrm{SpO}_{2}$ range within 94$96 \%$. In healthy adults aged older than 70 years, and who are non-smokers, the mean (SD) $\mathrm{SpO}_{2}$ is approximately $95 \%(1.5 \%)$, and healthy adults without obstructive sleep apnea have a mean minimum $\mathrm{SpO}_{2}$ of $90 \%$ during sleep $(31,32)$. Therefore, a target $\mathrm{SpO}_{2}$ lower limit of $94 \%$ is below the expected $\mathrm{SpO}_{2}$ of almost all healthy older adults who are awake, and above the mean minimum $\mathrm{SpO}_{2}$ when asleep. Furthermore, a previous study showed that the prevalence of hyperoxemia appeared to be negligible as long as the upper limit of $\mathrm{SpO}_{2}$ did not exceed $96 \%$ among critically ill patients (23). In addition, our results were 
similar to the British Thoracic Society (BTS) guideline recommended oxygen saturation target of $94-98 \%$ and the Australia and New Zealand Thoracic Society guideline recommended target of $92-96 \%$ except for in patients associated with chronic respiratory failure $(33,34)$. Another RCT in ICU patients suggested that a conservative protocol (maintaining $\mathrm{SpO}_{2}$ between 94\% and 98\%) for oxygen therapy compared with conventional therapy (maintaining $\mathrm{SpO}_{2}$ between $97 \%$ and $100 \%$ ) resulted in lower ICU and in-hospital mortality, which was consistent with our study (35). As pulse oximetry is widespread and affordable, implementation of the $94-96 \%$ target would be feasible, even in resource-limited environments.

Previously, at least six randomized controlled trials (RCTs) investigated the effect of administration of supplemental oxygen during AMI and concluded that oxygen therapy did not benefit patients with baseline normal peripheral oxygen saturations levels $\geq 90 \%$ (36-41). In addition, among critically ill patients, several studies suggested overuse of oxygen therapy is prevalent and is associated with adverse outcomes, including longer duration of mechanical ventilation and longer hospitalization $(42,43)$. However, these studies mainly focused on the comparison of clinical effect between routine oxygen therapy and ambient air, and did not explore the relationship between admission oxygen saturation and mortality. Similar to their findings, the univariable Cox analysis showed that oxygen therapy was not associated with mortality in our study. In addition, our subgroups analyses showed targeting $\mathrm{SpO}_{2}$ between $94 \%$ and $96 \%$ might optimize survival for patients with or without oxygen therapy. Additionally, in the AVOID trial, Stub et al. (38) randomized 441 patients with pre-hospital ST-elevation AMI to receive air or oxygen $(8 \mathrm{~L} / \mathrm{min}$ via mask) until discharge, and concluded that oxygen therapy may aggravate myocardial injury and was associated with increased myocardial infarct size (55\% larger) assessed at six months. At the end of treatment, $\mathrm{SpO}_{2}$ significantly differed between the oxygen group [100\% (IQR, 99-100\%)] and the ambient air group [98\% (IQR, 96-99\%); $\mathrm{P}<0.001]$, which suggested that the $\mathrm{SpO}_{2}$ in the ambient air group might be closer to the optimal $\mathrm{SpO}_{2}$ range we identified. Moreover, in prior randomized trials of oxygen therapy, the treatment group cut-off values for $\mathrm{SpO}_{2}$ were essentially arbitrary. Our study could provide a firmer basis for the selection of $\mathrm{SpO}_{2}$ targets within treatment groups for future research. Furthermore, while designing trials in this complex area, the method of oxygen delivery, levels, and duration of therapy should also be considered.
Several limitations of our study should be noted. Firstly, this study was a single-center retrospective observational study, and selection bias was inevitable. Thus, prospective cohorts are needed for further validation. Secondly, there were several potential confounding variables that we were unable to assess due to severe data missing conditions and other reasons. However, some of the excluded variables might have predictive value for clinical outcomes. Given that, external validation was required to test its utility. Finally, our study only focused on the in-hospital mortality of patients with AMI, while other outcomes, such as longterm mortality and late prognosis, were also important and deserved further investigation.

\section{Conclusions}

In a large and heterogeneous group of AMI patients, the relationship between admission $\mathrm{SpO}_{2}$ levels and in-hospital all-cause mortality followed a "U" shaped curve. The lowest mortality was observed for an $\mathrm{SpO}_{2}$ range of 94-96\%, and this finding could benefit future clinical trials of oxygen therapy.

\section{Acknowledgments}

Funding: This work was supported by the National Nature Science Foundation of China (No. 81770244), Medical Science and Technology Youth Cultivation Plan (No. 17QNP013 and No. 20QNPY038), Naval Military University Foreign Student Teaching Research and Reform Project (No. WJYA2018005), Shanghai Municipal Commission of Science and Technology (No. 17ZR1439100), Shanghai Shenkang Medicine Developing Project (No. SHDC12014107), and Shanghai Science and Technology Committee Medicine Leading Project (No. 15411960100).

\section{Footnote}

Reporting Checklist: The authors have completed the STROBE reporting checklist. Available at http://dx.doi. org/10.21037/atm-20-2614

Peer Review File: Available at http://dx.doi.org/10.21037/ atm-20-2614

Conflicts of Interest: All authors have completed the ICMJE uniform disclosure form (available at http://dx.doi. 
org/10.21037/atm-20-2614). The authors have no conflicts of interest to declare.

Ethical Statement: The authors are accountable for all aspects of the work in ensuring that questions related to the accuracy or integrity of any part of the work are appropriately investigated and resolved. The establishment of the Medical Information Mart for Intensive Care III (MIMIC-III) database is approved by the Institutional Review Boards (IRB) of the Massachusetts Institute of Technology (MIT, Cambridge, MA, USA) and the Beth Israel Deaconess Medical Center (BIDMC). Our study utilized the anonymous data available from this database, and hence the requirement for informed consent was waived. In summary, the study complied with the ethical standards laid down in the 1964 Declaration of Helsinki and its later amendments.

Open Access Statement: This is an Open Access article distributed in accordance with the Creative Commons Attribution-NonCommercial-NoDerivs 4.0 International License (CC BY-NC-ND 4.0), which permits the noncommercial replication and distribution of the article with the strict proviso that no changes or edits are made and the original work is properly cited (including links to both the formal publication through the relevant DOI and the license). See: https://creativecommons.org/licenses/by-nc-nd/4.0/.

\section{References}

1. Ibanez B, James S, Agewall S, et al. 2017 ESC Guidelines for the management of acute myocardial infarction in patients presenting with ST-segment elevation: The Task Force for the management of acute myocardial infarction in patients presenting with ST-segment elevation of the European Society of Cardiology (ESC). Eur Heart J 2018;39:119-77.

2. Piepoli MF, Hoes AW, Agewall S, et al. 2016 European Guidelines on cardiovascular disease prevention in clinical practice: The Sixth Joint Task Force of the European Society of Cardiology and Other Societies on Cardiovascular Disease Prevention in Clinical Practice (constituted by representatives of 10 societies and by invited experts) Developed with the special contribution of the European Association for Cardiovascular Prevention \& Rehabilitation (EACPR). Eur Heart J 2016;37:2315-81.

3. Hofmann R, James SK, Jernberg T, et al. Oxygen Therapy in Suspected Acute Myocardial Infarction. N Engl J Med 2017;377:1240-9.
4. Sjöberg F, Singer M. The medical use of oxygen: a time for critical reappraisal. J Intern Med 2013;274:505-28.

5. Moradkhan R, Sinoway LI. Revisiting the role of oxygen therapy in cardiac patients. J Am Coll Cardiol 2010;56:1013-6.

6. Zweier JL, Talukder MAH. The role of oxidants and free radicals in reperfusion injury. Cardiovasc Res 2006;70:181-90.

7. Jubran A. Pulse oximetry. Crit Care 2015;19:272.

8. Pretto JJ, Roebuck T, Beckert L, et al. Clinical use of pulse oximetry: official guidelines from the Thoracic Society of Australia and New Zealand. Respirology 2014;19:38-46.

9. James SK, Erlinge D, Herlitz J, et al. Effect of Oxygen Therapy on Cardiovascular Outcomes in Relation to Baseline Oxygen Saturation. JACC Cardiovasc Interv 2020;13:502-13.

10. Mueller C, Patrono C, Valgimigli M, et al. Questions and answers on diagnosis and risk assessment: a companion document of the 2015 ESC Guidelines for the management of acute coronary syndromes in patients presenting without persistent ST-segment elevation. Eur Heart J 2016;37:e15-e21.

11. Goldberger AL, Amaral LA, Glass L, et al. PhysioBank, PhysioToolkit, and PhysioNet: components of a new research resource for complex physiologic signals. Circulation 2000;101:E215-20.

12. Johnson AE, Pollard TJ, Shen L, et al. MIMIC-III, a freely accessible critical care database. Sci Data 2016;3:160035.

13. Thygesen K, Alpert JS, Jaffe AS, et al. Third universal definition of myocardial infarction. J Am Coll Cardiol 2012;60:1581-98.

14. Elixhauser A, Steiner C, Harris DR, et al. Comorbidity measures for use with administrative data. Med Care 1998;36:8-27.

15. White IR, Royston P, Wood AM. Multiple imputation using chained equations: Issues and guidance for practice. Stat Med 2011;30:377-99.

16. Zhang Z. Multiple imputation for time series data with Amelia package. Ann Transl Med 2016;4:56.

17. Fox J, Monette G. Generalized Collinearity Diagnostics. Publications of the American Statistical Association 1992;87:178-83.

18. Ahrens T, Tucker K. Pulse oximetry. Crit Care Nurs Clin North Am 1999;11:87-98.

19. Wukitsch MW, Petterson MT, Tobler DR, et al. Pulse oximetry: analysis of theory, technology, and practice. J Clin Monit 1988;4:290-301.

20. Allardet-Servent J, Sicard G, Metz V, et al. Benefits and 
risks of oxygen therapy during acute medical illness: Just a matter of dose! Rev Med Interne 2019;40:670-6.

21. Louie A, Feiner JR, Bickler PE, et al. Four Types of Pulse Oximeters Accurately Detect Hypoxia during Low Perfusion and Motion. Anesthesiology 2018;128:520-30.

22. van den Boom W, Hoy M, Sankaran J, et al. The Search for Optimal Oxygen Saturation Targets in Critically Ill Patients: Observational Data From Large ICU Databases. Chest 2020;157:566-73.

23. Durlinger EMJ, Spoelstra-de Man AME, Smit B, et al. Hyperoxia: At what level of $\mathrm{SpO} 2$ is a patient safe? A study in mechanically ventilated ICU patients. J Crit Care 2017;39:199-204.

24. Morrow DA, Antman EM, Charlesworth A, et al. TIMI risk score for ST-elevation myocardial infarction: A convenient, bedside, clinical score for risk assessment at presentation: An intravenous nPA for treatment of infarcting myocardium early II trial substudy. Circulation 2000;102:2031-7.

25. Fox KAA, Dabbous OH, Goldberg RJ, et al. Prediction of risk of death and myocardial infarction in the six months after presentation with acute coronary syndrome: prospective multinational observational study (GRACE). BMJ 2006;333:1091-4.

26. Faulhaber $M$, Wille $M$, Gatterer H, et al. Resting arterial oxygen saturation and breathing frequency as predictors for acute mountain sickness development: a prospective cohort study. Sleep Breath 2014;18:669-74.

27. Zorlu A, Bektasoglu G, Guven FM, et al. Usefulness of admission red cell distribution width as a predictor of early mortality in patients with acute pulmonary embolism. Am J Cardiol 2012;109:128-34.

28. Xie H, Su H, Chen D, et al. Use of Autofluorescence to Intraoperatively Diagnose Visceral Pleural Invasion From Frozen Sections in Patients With Lung Adenocarcinoma 2 cm or Less. Am J Clin Pathol 2019;152:608-15.

29. Walton RAL, Hansen BD. Venous oxygen saturation in critical illness. J Vet Emerg Crit Care (San Antonio) 2018;28:387-97.

30. Huang SS, Chen Y-H, Lu T-M, et al. Application of the Sequential Organ Failure Assessment score for predicting mortality in patients with acute myocardial infarction. Resuscitation 2012;83:591-5.

31. Hardie JA, Vollmer WM, Buist AS, et al. Reference values for arterial blood gases in the elderly. Chest 2004;125:2053-60.

32. Gries RE, Brooks LJ. Normal Oxyhemoglobin Saturation During Sleep. Chest 1996;110:1489-92.
33. Beasley R, Chien J, Douglas J, et al. Thoracic Society of Australia and New Zealand oxygen guidelines for acute oxygen use in adults: 'Swimming between the flags'. Respirology 2015;20:1182-91.

34. O'Driscoll BR, Howard LS, Davison AG. BTS guideline for emergency oxygen use in adult patients. Thorax 2008;63 Suppl 6:vi1-vi68.

35. Girardis M, Busani S, Damiani E, et al. Effect of Conservative vs Conventional Oxygen Therapy on Mortality Among Patients in an Intensive Care Unit. JAMA 2016;316:1583-9.

36. Abuzaid A, Fabrizio C, Felpel K, et al. Oxygen Therapy in Patients with Acute Myocardial Infarction: A Systemic Review and Meta-Analysis. Am J Med 2018;131:693-701.

37. Sepehrvand N, James SK, Stub D, et al. Effects of supplemental oxygen therapy in patients with suspected acute myocardial infarction: a meta-analysis of randomised clinical trials. Heart 2018;104:1691-8.

38. Stub D, Smith K, Bernard S, et al. Air Versus Oxygen in ST-Segment-Elevation Myocardial Infarction. Circulation 2015;131:2143-50.

39. Ukholkina GB, Kostianov IIu, Kuchkina NV, et al. Effect of oxygenotherapy used in combination with reperfusion in patients with acute myocardial infarction. Kardiologiia 2005;45:59.

40. Khoshnood A, Carlsson M, Akbarzadeh M, et al. Effect of oxygen therapy on myocardial salvage in ST elevation myocardial infarction: The randomized SOCCER trial. Eur J Emerg Med 2018;25:78-84.

41. Ranchord AM, Argyle R, Beynon R, et al. Highconcentration versus titrated oxygen therapy in STelevation myocardial infarction: a pilot randomized controlled trial. Am Heart J 2012;163:168-75.

42. Itagaki T, Nakano $\mathrm{Y}$, Okuda $\mathrm{N}$, et al. Hyperoxemia in mechanically ventilated, critically ill subjects: incidence and related factors. Respir Care 2015;60:335-40.

43. Suzuki S, Eastwood GM, Peck L, et al. Current oxygen management in mechanically ventilated patients: a prospective observational cohort study. J Crit Care 2013;28:647-54.

Cite this article as: Yu Y, Wang J, Wang Q, Wang J, Min J, Wang S, Wang P, Huang R, Xiao J, Zhang Y, Wang Z. Admission oxygen saturation and all-cause in-hospital mortality in acute myocardial infarction patients: data from the MIMICIII database. Ann Transl Med 2020;8(21):1371. doi: 10.21037/atm20-2614 